\title{
Pressure-Based and Potential-Based Differential Quadrature Procedures for Free Vibration of Circular Plates in Contact with Fluid
}

\section{Abstract}

The differential quadrature method (DQM) has been so far applied to a wide variety of fluid and/or structural problems. The results of many researchers reveal that the DQM is computationally efficient and is applicable to a large class of boundary value problems. However, there is little information about its applications to fluidstructure interaction problems. Therefore, the purpose of this paper is to provide some information in this area and to develop procedures based on the DQM for the numerical solution of fluidstructure interaction problems. First, the governing partial differential equations of motion of the structure and fluid are discretized separately using the DQM. Then, by applying the boundary condition at fluid-structure interface, the governing eigenvalue equations of the coupled system are obtained which can then be solved for the eigenvalues of the system. The applicability of the proposed procedures is shown herein through the free vibration analysis of thin circular plates in contact with a cylindrical fluid-filled cavity. Issues related to the implementation of the regularity conditions at the center of the circular plate and the central line of the cylindrical cavity are addressed. Two new regularity conditions are proposed for the circular cylindrical fluid domain. The accuracy and efficiency of the proposed procedures are demonstrated by comparing the obtained results with those available in the literature. It is shown that highly accurate converged results can be obtained by the proposed procedures using a small number of grid points. Three new dimensionless parameters and variables are also introduced for the free vibration of the coupled system. The influences of these parameters on dynamic behavior of the system are studied.

\section{Keywords}

$D Q M$, fluid-structure interaction, pressure-based DQM, potential-based DQM, circular plates, cylindrical cavity.

\section{S. A. Eftekhari *}

Young Researchers and Elite Club, Karaj Branch, Islamic Azad University, Karaj, P.O. Box 31485-313, Iran

*Author email:

aboozar.eftekhari@gmail.com

http://dx.doi.org/10.1590/1679-78252321

Received 30.07.2015

In revised form 07.11.2015

Accepted 04.01.2016

Available online 05.01.2016 


\section{INTRODUCTION}

The problem of plate-like structures interacting with a fluid has been an interesting research subject for a long time because this problem can be found in many applications such as offshore structures, nuclear reactors, micro pumps, storage tanks in airplanes and space vehicles and the like. Therefore, it is of great importance to accurately predict the dynamic behavior and vibration characteristics of such structures.

Various analytical and numerical methods have been developed in the past to study the behavior of plate-like structures interacting with a fluid. But most of the research efforts mainly focus on the free vibration analysis of plates in contact with an incompressible fluid. Kwak and Kim (1991) proposed an analytical procedure using the integral transformation technique in conjunction with the dual integration equation method to study the effect of fluid on the natural frequencies of circular plates vibrating axisymmetrically in contact with an incompressible fluid. They also introduced the concept of non-dimensionalized added virtual mass incremental (NAVMI) factor for fluid-loaded circular plates that reflects the increase of inertia due to the presence of the fluid. Bauer (1995) developed an analytical procedure to determine the coupled hydroelastic natural frequencies of circular membranes and plates backed by a cylindrical fluid-filled cavity. The fluid was assumed to be incompressible and frictionless. Amabili and Kwak (1999) proposed an analytical formulation using the perturbation technique in conjunction with the Hankel transformation method to investigate effects of free-surface waves on free vibrations of circular plates resting on a free fluid surface. In their study, the fluid was considered inviscid and incompressible. They showed that the effect of free-surface waves on the plate's natural frequencies is significant only when the bulging and sloshing modes of the system have close natural frequencies. Jeong and Kim (2005) developed an analytical algorithm by the Rayleigh-Ritz approach based on the Fourier-Bessel series expansion for the linear free vibration of a circular plate submerged in a bounded compressible fluid. They also solved the problem using the finite element method and studied the effects of fluid compressibility on natral frequencies of the coupled system. Chiba (1994), Amabili et al. (1995), Kwak (1997), Kwak and Han (2000), Jeong (2003), Ergin and Ugurlu (2004), Kim and Lee (2005), Gorman and Horáček (2007), Gorman et al. (2008), Jeong et al. (2009), Askari and Daneshmand (2010), Askari et al. (2013), Tariverdilo et al. (2013), and Shafiee et al. (2014) also solved various circular plate-fluid interaction problems using various analytical or approximate methods.

The differential quadrature method (DQM), which was firstly introduced by Bellman and his associates $(1971,1972)$ in the early 1970s, is a powerful numerical method for the direct solution of partial differential equations that arise in various fields of engineering, mathematics, and physics (Bert and Malik, 1996; Tornabene et al., 2015). It is simple to use and also straightforward to implement. Since its introduction, the DQM has been successfully applied to a wide variety of fluid and/or structural problems (Shu and Richards, 1992; Shu et al., 1995; Bert and Malik, 1996; Shu and Chew, 1999; Shu, 2000; Liu and Wu, 2001; Eftekhari and Jafari 2013; Fantuzzi et al., 2014). However, there are few studies on its applications to fluid-structure interaction problems.

In the literature, one may refer to the work done by Koohkan et al. (2010) or the recent studies by Eftekhari and Jafari (2014) and Eftekhari (2016). Koohkan et al. (2010) proposed a combined application of the DQM and the finite difference method to study the transient responses of damreservoir systems under ground motion. In their study, the dam structure has been modeled as an 
Euler-Bernoulli beam while the reservoir modeled as a semi-infinite fluid. Eftekhari and Jafari (2014) proposed a mixed modal-DQM formulation to study the free and forced vibrations of beams in contact with fluid. It has been claimed that the mixed method can produce much better accuracy than the finite element method (FEM) using less computational time. Most recently, Eftekhari (2015) proposed a coupled Ritz-DQM formulation for the dynamic analysis of Timoshenko beams in contact with fluid. It has been concluded that the conditioning of the resulting eigenvalue (or ordinary differential) equations of the potential-based formulation is considerably better than that of the pressure-based formulation. As it can be seen from above literature survey, all of abovementioned works are limited to beam-fluid interaction problems. Besides, the formulations in Eftekhari and Jafari (2014) and Eftekhari (2015) require the help of another method (modal technique or Ritz method) to handle the fluid-structure interaction problem. Therefore, the purpose of this paper is to provide some more information in this area and to develop general procedures based on the DQM for the numerical solution of fluid-structure interaction problems.

In this study, two general procedures based on the DQM, i.e., the pressure-based and potentialbased DQMs, are proposed to study the free vibration of thin circular plates in contact with a bounded compressible fluid. First, the governing partial differential equation of motion of the circular plate and that of the fluid are discretized separately using the DQM. Then, by applying the boundary condition at fluid-structure interface, the governing eigenvalue equations of the coupled system are obtained which can then be solved for the eigenvalues of the system. The proposed procedures, in general, enjoy from simplicity and high accuracy of the DQM. Their reliability and efficiency are shown through numerical simulations.

\section{DQM}

Let $w(\zeta)$ be an arbitrary function and $\zeta_{1}, \zeta_{2}, \ldots, \zeta_{n}$ be a set of grid points in the $\zeta$-direction. According to the DQM, the qth-order derivative of the function $w(\zeta)$ at any grid point can be approximated by the following formulation

$$
w^{(q)}\left(\zeta_{i}\right)=\sum_{j=1}^{n} A_{i j}^{(q)} w\left(\zeta_{j}\right)
$$

where $\mathrm{w}\left(\zeta_{\mathrm{j}}\right)$ represents the function value at a grid point $\zeta_{\mathrm{j}}$ and $A_{i j}^{(q)}$ are the weighting coefficients of the qth-order derivative. The first-order weighting coefficients, i.e., $A_{i j}^{(1)}$, are given by (Quan and Chang, 1989)

$$
A_{i k}^{(1)}=\left\{\begin{array}{ccc}
\frac{\Pi\left(\zeta_{i}\right)}{\left(\zeta_{i}-\zeta_{k}\right) \Pi\left(\zeta_{k}\right)} & i \neq k, & i, k=1,2, \ldots, n \\
-\sum_{j=1, j \neq i}^{n} A_{i j}^{(1)} & i=k, & i=1,2, \ldots, n
\end{array}\right.
$$

where

$$
\Pi\left(\zeta_{i}\right)=\prod_{j=1, j \neq i}^{n}\left(\zeta_{i}-\zeta_{j}\right)
$$


The weighting coefficients of the higher-order derivatives can be computed from the following recurrence relationship (Bert and Malik, 1996)

$$
[\boldsymbol{A}]^{(q)}=[\boldsymbol{A}]^{(q-1)}[\boldsymbol{A}]^{(1)}=[\boldsymbol{A}]^{(1)}[\boldsymbol{A}]^{(q-1)}, q>1
$$

In this work, the DQM grid points are taken nonuniformly spaced and are given by the following equations (Bert and Malik, 1996)

$$
\zeta_{i}=\frac{1}{2}\left[1-\cos \left(\frac{(i-1) \pi}{n-1}\right)\right], i=1,2,3, \ldots, n, 0 \leq \zeta \leq 1
$$

\section{GOVERNING EQUATIONS AND BOUNDARY CONDITIONS}

The geometry and coordinate system for a circular thin plate backed by a cylindrical fluid-filled cavity is shown schematically in Figure 1. The circular cylindrical cavity of diameter $2 a$ and height $H$ is filled with a compressible and inviscid fluid of density @f. The cavity bottom at $z=H$ and the side wall at $r=a$ are considered as rigid walls, while the fluid surface at $z=0$ is covered with a flexible circular plate. The fluid motion is assumed to be irrotational and of small amplitude. The governing partial differential equations of the coupled system can be expressed in terms of different variables of the fluid such as displacement, pressure, and velocity potential. In this study, we are interested to describe them in terms of pressure and velocity potential variables. The details are given in the following subsections.

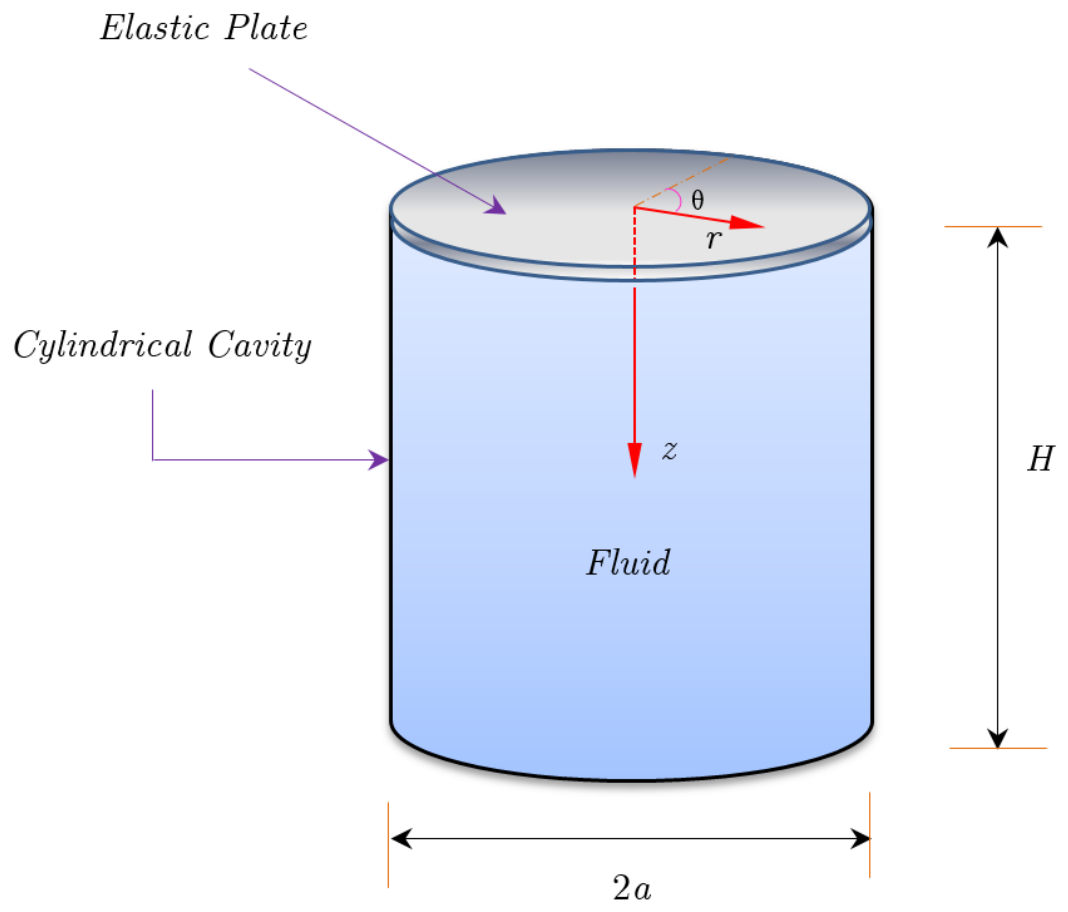

Figure 1: Geometry and coordinate system for a circular plate in contact with a fluid-filled cylindrical cavity. 


\subsection{Governing Equations and Boundary Conditions for Pressure-Based Procedure}

When pressure variable is used to formulate the present fluid-structure interaction problem, the governing partial differential equations of the coupled system can be expressed as

$$
D \nabla^{4} w(r, \theta, t)+\varrho_{s} h \frac{\partial^{2} w(r, \theta, t)}{\partial t^{2}}=-p(r, \theta, z=0, t)
$$

and

$$
\nabla^{2} p(r, \theta, z, t)+\frac{\partial^{2} p(r, \theta, z, t)}{\partial z^{2}}=\frac{1}{c_{f}^{2}} \frac{\partial^{2} p(r, \theta, z, t)}{\partial t^{2}}
$$

where $D$ is bending (or flexural) stiffness of the plate, $w(r, \theta, t)$ is the deflection of the plate, $\varrho_{s}$ is the mass density of the structure (plate), $h$ is thickness of the plate, $p(r, \theta, z, t)$ is the hydrodynamic pressure distributions in excess of the hydrostatic pressure, and $c_{f}$ is the speed of sound in fluid. Furthermore,

$$
\begin{gathered}
\nabla^{2}=\frac{\partial^{2}}{\partial r^{2}}+\frac{1}{r} \frac{\partial}{\partial r}+\frac{1}{r^{2}} \frac{\partial^{2}}{\partial \theta^{2}} \\
\nabla^{4}=\nabla^{2} \nabla^{2}=\left(\frac{\partial^{2}}{\partial r^{2}}+\frac{1}{r} \frac{\partial}{\partial r}+\frac{1}{r^{2}} \frac{\partial^{2}}{\partial \theta^{2}}\right)\left(\frac{\partial^{2}}{\partial r^{2}}+\frac{1}{r} \frac{\partial}{\partial r}+\frac{1}{r^{2}} \frac{\partial^{2}}{\partial \theta^{2}}\right)= \\
\frac{\partial^{4}}{\partial r^{4}}+\frac{2}{r} \frac{\partial^{3}}{\partial r^{3}}-\frac{1}{r^{2}}\left(\frac{\partial^{2}}{\partial r^{2}}-2 \frac{\partial^{4}}{\partial r^{2} \partial \theta^{2}}\right)+\frac{1}{r^{3}}\left(\frac{\partial}{\partial r}-2 \frac{\partial^{3}}{\partial r \partial \theta^{2}}\right)+\frac{1}{r^{4}}\left(4 \frac{\partial^{2}}{\partial \theta^{2}}+\frac{\partial^{4}}{\partial \theta^{4}}\right)
\end{gathered}
$$

In this study, the free vibration of fluid-loaded circular plates with clamped edge is considered. The boundary conditions of the plate for this case are (Rao, 2007)

$$
w(r=a, \theta, t)=\frac{\partial w(r=a, \theta, t)}{\partial r}=0
$$

The boundary conditions of the fluid cavity are assumed to be in the form of Neumann-type boundary conditions. In other words,

$$
\frac{\partial p(r=a, \theta, z, t)}{\partial r}=\frac{\partial p(r, \theta, z=H, t)}{\partial z}=0, \frac{\partial p(r, \theta, z=0, t)}{\partial z}=-\varrho_{f} \frac{\partial^{2} w(r, \theta, t)}{\partial t^{2}}
$$

where $\varrho$ is the mass density of the fluid. For free vibration of the coupled system, the variables $w$ and $p$ are assumed to be harmonic as

$$
w(r, \theta, t)=\widetilde{w}(r, \theta) e^{i \omega t}, \quad p(r, \theta, z, t)=\widetilde{p}(r, \theta, z) e^{i \omega t}
$$

where $\omega$ is the circular frequency. It is advantageous to introduce the following parameters and variables 


$$
\lambda=\frac{\varrho_{f} a}{\varrho_{s} h}, \vartheta=\frac{H}{a}, c=\frac{c_{f}}{c_{s}}, c_{s}=\frac{1}{a} \sqrt{\frac{D}{\varrho_{s} h}}, P=\frac{a^{4}}{D} \tilde{p}, W=\widetilde{w}, \quad R=\frac{r}{a}, \quad Z=\frac{z}{H}, \quad \Omega=\omega a^{2} \sqrt{\frac{\varrho_{s} h}{D}},
$$

where $\lambda$ is the mass ratio, $\vartheta$ is the slenderness ratio, $c$ is the speed ratio, $c s$ is the speed of sound in structure (circular plate), and $\Omega$ is the dimensionless natural frequency of the coupled system. Now, substituting Eqs. (12) and (13) into Eqs. (6) and (7) gives

$$
\nabla^{4} W(R, \theta)-\Omega^{2} W(R, \theta)=-P(R, \theta, Z=0)
$$

and

$$
\nabla^{2} P(R, \theta, Z)+\frac{1}{\vartheta^{2}} \frac{\partial^{2} P(R, \theta, Z)}{\partial Z^{2}}=-\frac{1}{c^{2}} \Omega^{2} P(R, \theta, Z)
$$

Consequently, the boundary conditions of the fluid cavity becomes

$$
\frac{\partial P(R=1, \theta, Z)}{\partial R}=\frac{\partial p(R, \theta, Z=1)}{\partial Z}=0, \frac{\partial P(R, \theta, Z=0)}{\partial Z}=\lambda \vartheta \Omega^{2} W(R, \theta)
$$

It can be clearly seen from Eqs. (14)-(16) that the natural frequencies of the coupled system can be characterized by means of the following three dimensionless parameters

$$
\lambda=\frac{\varrho_{f} a}{\varrho_{s} h}, \vartheta=\frac{H}{a}, c=\frac{c_{f}}{c_{s}}
$$

\subsection{Governing Equations and Boundary Conditions for Potential-Based Procedure}

When velocity potential variable is used to formulate the present fluid-structure interaction problem, the governing partial differential equations of the coupled system can be expressed as

$$
D \nabla^{4} w(r, \theta, t)+\varrho_{s} h \frac{\partial^{2} w(r, \theta, t)}{\partial t^{2}}=\varrho_{f} \frac{\partial \varphi(r, \theta, z=0, t)}{\partial t}
$$

and

$$
\nabla^{2} \varphi(r, \theta, z, t)+\frac{\partial^{2} \varphi(r, \theta, z, t)}{\partial z^{2}}=\frac{1}{c_{f}^{2}} \frac{\partial^{2} \varphi(r, \theta, z, t)}{\partial t^{2}}
$$

where $\varphi(r, \theta, t)$ is the velocity potential of the fluid. In this case, the boundary conditions of the fluid cavity are

$$
\frac{\partial \varphi(r=a, \theta, z, t)}{\partial r}=\frac{\partial \varphi(r, \theta, z=H, t)}{\partial z}=0, \frac{\partial \varphi(r, \theta, z=0, t)}{\partial z}=\frac{\partial w(r, \theta, t)}{\partial t}
$$

For free vibration of the coupled system, the variables $w$ and $\varphi$ are assumed to be harmonic as

$$
w(r, \theta, t)=\widetilde{w}(r, \theta) e^{\omega t}, \varphi(r, \theta, z, t)=\widetilde{\varphi}(r, \theta, z) e^{\omega t}
$$


Introducing Eqs. (13), (21) and

$$
\Theta=\frac{\varrho_{f} a^{2}}{\sqrt{\varrho_{s} h D}} \widetilde{\varphi}
$$

to Eqs. (18)-(20) gives

$$
\begin{gathered}
\nabla^{4} W(R, \theta)+\Omega^{2} W(R, \theta)=\Omega \Theta(R, \theta, Z=0) \\
\nabla^{2} \Theta(R, \theta, Z)+\frac{1}{\vartheta^{2}} \frac{\partial^{2} \Theta(R, \theta, Z)}{\partial Z^{2}}=\frac{1}{c^{2}} \Omega^{2} \Theta(R, \theta, Z) \\
\frac{\partial \Theta(R=1, \theta, Z)}{\partial R}=\frac{\partial \Theta(R, \theta, Z=1)}{\partial Z}=0, \frac{\partial \Theta(R, \theta, Z=0)}{\partial Z}=\lambda \vartheta \Omega W(R, \theta)
\end{gathered}
$$

At this stage, the DQM can be employed to discretize the dimensionless differential equations of the fluid and plate. However, there are some important issues related to regularity conditions at $R$ $=0$ (or at $r=0$ ) that need to be addressed first. The details on regularity conditions are given in the following section.

\section{REGULARITY CONDITIONS AT R $=0$}

It can be seen from Eqs. (14) and (15) (or Eqs. 23 and 24) that the governing partial differential equation of the circular plate is of order four with respect to $R$ coordinate variable while that of the fluid is of order two with respect to this coordinate variable. The motion of the plate in $R$-direction is also subjected to two boundary conditions (see Eq. 10) while that of the fluid is subjected to one boundary condition (see Eq. 16 or 25). It is well known that an arbitrary qth-order differential equation should involve $q$ number of boundary conditions. In the present problem, however, the number of boundary conditions is smaller than the order of differential equation for both the circular plate and circular cylindrical fluid-filled cavity. Therefore, difficulty arises when trying to solve such type of problem using numerical algorithms like the DQM.

The above-mentioned difficulty has also been reported before when solving similar problems using the DQM. For instance, in solving the free vibration problem of circular plates, Wu and Liu (2001) also Wu et al. (2002) have also addressed this difficulty. They pointed out that some regularity conditions should be applied at the plate center in order to solve such type of problems. They assumed the deflection of the plate in the form

$$
W(R, \theta)=\sum_{k=0}^{\infty} W_{k}(R) \cos (k \theta)
$$

and proposed the following regularity conditions

$$
W_{k}(R=0)=\frac{\mathrm{d}^{2} W_{k}(R=0)}{\mathrm{d} R^{2}}=0, \quad k=1,3,5,7, \ldots
$$




$$
\frac{\mathrm{d} W_{k}(R=0)}{\mathrm{d} R}=\frac{\mathrm{d}^{3} W_{k}(R=0)}{\mathrm{d} R^{3}}=0, \quad k=0,2,4,6, \ldots
$$

The numerical results presented in $\mathrm{Wu}$ and $\mathrm{Liu}$ (2001) and $\mathrm{Wu}$ et al. (2002) showed that the above regularity conditions can give accurate solutions for natural frequencies of circular plates. In this study, the above regularity conditions are applied at the center of the fluid-loaded circular plate. In addition, some regularity conditions should also be applied at the center line of the circular cylindrical fluid-filled cavity. Let

$$
P(R, \theta, Z)=\sum_{k=0}^{\infty} P_{k}(R, Z) \cos (k \theta), \Theta(R, \theta, Z)=\sum_{k=0}^{\infty} \Theta_{k}(R, Z) \cos (k \theta)
$$

The following regularity conditions are introduced for the fluid domain

$$
\begin{gathered}
P_{k}(R=0, Z)=\Theta_{k}(R=0, Z)=0, \quad k=1,3,5,7, \ldots \\
\frac{\partial P_{k}(R=0, Z)}{\partial R}=\frac{\partial \Theta_{k}(R=0, Z)}{\partial R}=0, \quad k=0,2,4,6, \ldots
\end{gathered}
$$

Our numerical experiments showed that the above regularity conditions can produce accurate solutions for natural frequencies of fluid-loaded circular plates.

\section{PRESSURE-BASED DQM PROCEDURE}

The governing partial differential equations for free vibration of the coupled system are given in Eqs. (14) and (15). The solutions to these equations are first assumed to be in the forms of Eqs. (26) and (29). Substituting Eqs. (26) and (29) into Eqs. (14) and (15) gives

$$
\begin{gathered}
{\left[\frac{\mathrm{d}^{4}}{\mathrm{~d} R^{4}}+\frac{2}{R} \frac{\mathrm{d}^{3}}{\mathrm{~d} R^{3}}-\frac{1+2 k^{2}}{R^{2}} \frac{\mathrm{d}^{2}}{\mathrm{~d} R^{2}}+\frac{1+2 k^{2}}{R^{3}} \frac{\mathrm{d}}{\mathrm{d} R}+\frac{k^{4}-4 k^{2}}{R^{4}}\right] W_{k}(R)-\Omega^{2} W_{k}(R)=-P_{k}(R, Z=0)} \\
{\left[\frac{\partial^{2}}{\partial R^{2}}+\frac{1}{R} \frac{\partial}{\partial R}-\frac{k^{2}}{R^{2}}+\frac{1}{\vartheta^{2}} \frac{\partial^{2}}{\partial Z^{2}}\right] P_{k}(R, Z)+\frac{1}{c^{2}} \Omega^{2} P_{k}(R, Z)=0}
\end{gathered}
$$

At this stage, the DQM will be used to discretize Eqs. (32) and (33). The details are given in the following subsections.

\subsection{Discretization of Governing Differential Equation of the Structure}

Consider $n$ grid points with coordinates $R_{1}, R_{2}, \ldots, R_{n}$ in the $R$-direction. Satisfying Eq. (32) at any grid point $R=R_{i}(i=1,2, \ldots, n)$ and substituting the quadrature rule into results gives

$$
\left[\boldsymbol{K}_{k}^{s}\right]\left\{\boldsymbol{W}_{k}\right\}-\Omega^{2}\left[\boldsymbol{M}^{s}\right]\left\{\boldsymbol{W}_{k}\right\}=-\left\{\boldsymbol{P}_{k}^{I}\right\}
$$

where $\left[\boldsymbol{M}^{s}\right]$ and $\left[\boldsymbol{K}_{k}^{s}\right]$ are structural mass and stiffness matrices, $\left\{\boldsymbol{W}_{k}\right\}$ is the displacement vector, and $\left\{\boldsymbol{P}_{k}^{I}\right\}$ is the load vector due to fluid-structure interaction. $\left[\boldsymbol{M}^{s}\right],\left[\boldsymbol{K}_{k}^{s}\right],\left\{\boldsymbol{W}_{k}\right\}$ and $\left\{\boldsymbol{P}_{k}^{I}\right\}$ are given by 


$$
\begin{aligned}
& {\left[\boldsymbol{M}^{s}\right]=[\boldsymbol{I}]} \\
& {\left[\boldsymbol{K}_{k}^{s}\right]=[\boldsymbol{A}]^{(4)}+2[\boldsymbol{R}]^{(1)}[\boldsymbol{A}]^{(3)}-\left(1+2 k^{2}\right)[\boldsymbol{R}]^{(2)}[\boldsymbol{A}]^{(2)}+\left(1+2 k^{2}\right)[\boldsymbol{R}]^{(3)}[\boldsymbol{A}]^{(1)}+\left(k^{4}-4 k^{2}\right)[\boldsymbol{R}]^{(4)}} \\
& {[\boldsymbol{R}]^{(1)}=\operatorname{diag}\{\boldsymbol{R}\}^{(1)}, \quad\{\boldsymbol{R}\}^{(1)}=\left\{\begin{array}{llll}
\frac{1}{R_{1}} & \frac{1}{R_{2}} & \ldots & \frac{1}{R_{n}}
\end{array}\right\}^{T}} \\
& {[\boldsymbol{R}]^{(2)}=\operatorname{diag}\{\boldsymbol{R}\}^{(2)}, \quad\{\boldsymbol{R}\}^{(2)}=\left\{\begin{array}{llll}
\frac{1}{R_{1}^{2}} & \frac{1}{R_{2}^{2}} & \ldots & \frac{1}{R_{n}^{2}}
\end{array}\right\}^{T}} \\
& {[\boldsymbol{R}]^{(3)}=\operatorname{diag}\{\boldsymbol{R}\}^{(3)}, \quad\{\boldsymbol{R}\}^{(3)}=\left\{\begin{array}{llll}
\frac{1}{R_{1}^{3}} & \frac{1}{R_{2}^{3}} & \ldots & \frac{1}{R_{n}^{3}}
\end{array}\right\}^{T}} \\
& {[\boldsymbol{R}]^{(4)}=\operatorname{diag}\{\boldsymbol{R}\}^{(4)}, \quad\{\boldsymbol{R}\}^{(4)}=\left\{\begin{array}{llll}
\frac{1}{R_{1}^{4}} & \frac{1}{R_{2}^{4}} & \ldots & \frac{1}{R_{n}^{4}}
\end{array}\right\}^{T}} \\
& \left\{\boldsymbol{W}_{k}\right\}=\left\{\begin{array}{llll}
W_{k}\left(R_{1}\right) & W_{k}\left(R_{2}\right) & \ldots & W_{k}\left(R_{n}\right)
\end{array}\right\}^{T} \\
& \left\{\boldsymbol{P}_{k}^{I}\right\}=\left\{\begin{array}{llll}
P_{k}\left(R_{1}, 0\right) & P_{k}\left(R_{2}, 0\right) & \ldots & P_{k}\left(R_{n}, 0\right)
\end{array}\right\}^{T}
\end{aligned}
$$

where in $[\boldsymbol{I}]$ is an identity matrix of order $n \times n$, and $[\boldsymbol{A}]^{(q)}(q=1,2,3,4)$ are the DQM weighting coefficient matrix of the $q$ th-order $R$-derivative.

\subsection{Discretization of Governing Differential Equation of the Fluid}

Consider $n$ grid points with coordinates $R_{1}, R_{2}, \ldots, R_{n}$ in the $R$-direction and $m$ grid points with coordinates $Z_{1}, Z_{2}, \ldots, Z_{m}$ in the $Z$-direction. Satisfying Eq. (33) at any grid point $R=R_{i}(i=1,2$, $\ldots, n)$ and substituting the quadrature rule into results gives

$$
\left[[\boldsymbol{A}]^{(2)}+[\boldsymbol{R}]^{(1)}[\boldsymbol{A}]^{(1)}-k^{2}[\boldsymbol{R}]^{(2)}+\frac{1}{\vartheta^{2}}[\boldsymbol{I}] \frac{\partial^{2}}{\partial Z^{2}}\right]\left\{\boldsymbol{P}_{k}(Z)\right\}+\frac{1}{c^{2}} \Omega^{2}[\boldsymbol{I}]\left\{\boldsymbol{P}_{k}(Z)\right\}=\{\mathbf{0}\}
$$

where in $\{0\}$ is a zero vector of order $n \times 1$, and

$$
\left\{\boldsymbol{P}_{k}(Z)\right\}=\left\{P_{k}\left(R_{1}, Z\right) \quad P_{k}\left(R_{2}, Z\right) \quad \ldots \quad P_{k}\left(R_{n}, Z\right)\right\}^{T}
$$

Now, satisfying Eq. (43) at any grid point $Z=Z_{i}(i=1,2, \ldots, m)$ and using the quadrature rule yields

$$
\left[\boldsymbol{K}_{k}^{f}\right]\left\{\boldsymbol{P}_{k}\right\}+\Omega^{2}\left[\boldsymbol{M}^{f}\right]\left\{\boldsymbol{P}_{k}\right\}=\{\tilde{\mathbf{0}}\}
$$

where $\left[\boldsymbol{M}^{f}\right]$ and $\left[\boldsymbol{K}_{k}^{f}\right]$ are the mass and stiffness matrices of the fluid, $\left\{\boldsymbol{P}_{k}\right\}$ is the vector of pressure values, and $\{\widetilde{\mathbf{0}}\}$ is a zero vector of order $\mathrm{nm} \times 1$. The $\mathrm{n} \times \mathrm{n}$ sub-matrices $\left[\boldsymbol{M}_{i j}^{f}\right]$ and $\left[\boldsymbol{K}_{k, i j}^{f}\right]$ are given by 


$$
\begin{gathered}
{\left[\boldsymbol{M}_{i j}^{f}\right]=\frac{1}{c^{2}} \bar{I}_{i j}[\boldsymbol{I}], \quad i, j=1,2, \ldots, m} \\
{\left[\boldsymbol{K}_{k, i j}^{f}\right]=\bar{I}_{i j}\left([\boldsymbol{A}]^{(2)}+[\boldsymbol{R}]^{(1)}[\boldsymbol{A}]^{(1)}-k^{2}[\boldsymbol{R}]^{(2)}\right)+\frac{1}{\vartheta^{2}} \bar{A}_{i j}^{(2)}[\boldsymbol{I}]}
\end{gathered}
$$

where $\bar{I}_{i j}$ are the elements of $m \times m$ identity matrix, and $\bar{A}_{i j}^{(2)}$ are the DQM weighting coefficients of the second-order Z-derivative. Furthermore,

$$
\left\{\boldsymbol{P}_{k}\right\}=\left\{\left\{\boldsymbol{P}_{k}\left(Z_{1}\right)\right\}^{T} \quad\left\{\boldsymbol{P}_{k}\left(Z_{2}\right)\right\}^{T} \quad \ldots \quad\left\{\boldsymbol{P}_{k}\left(Z_{m}\right)\right\}^{T}\right\}^{T}
$$

where in the vector $\left\{\boldsymbol{P}_{k}(Z)\right\}$ is defined in Eq. (44).

\subsection{Implementation of Boundary and Regularity Conditions}

There are different approaches to incorporate the boundary conditions in the DQM resultant matrix equations. The technique recently proposed by the present author (Eftekhari, 2015) is used here to implement the boundary conditions of the problem. According to this new approach, the quadrature analog equations of the boundary conditions are first directly substituted in the resultant matrix equations of the DQM. The degrees of freedom correspond to the Dirichlet-type boundary equations are then removed from the final matrix equations.

\subsubsection{Quadrature Analogs of the Boundary and Regularity Conditions of the Circular Plate}

The boundary and regularity conditions of the circular plate are given in Eqs. (10), (27) and (28). The quadrature analogs of the plate boundary conditions are simply

$$
\begin{gathered}
W_{k}\left(R_{n}\right)=0, \quad k=1,2,3,4, \ldots \\
\sum_{j=1}^{n} A_{n j}^{(1)} W_{k}\left(R_{j}\right)=0, \quad k=1,2,3,4, \ldots
\end{gathered}
$$

Similarly, the quadrature analogs of the regularity conditions are obtained as

$$
\begin{gathered}
W_{k}\left(R_{1}\right)=\sum_{j=1}^{n} A_{1 j}^{(2)} W_{k}\left(R_{j}\right)=0, \quad k=1,3,5,7, \ldots \\
\sum_{j=1}^{n} A_{1 j}^{(1)} W_{k}\left(R_{j}\right)=\sum_{j=1}^{n} A_{1 j}^{(3)} W_{k}\left(R_{j}\right)=0, \quad k=0,2,4,6, \ldots
\end{gathered}
$$

\subsubsection{Quadrature Analogs of the Boundary and Regularity Conditions of the Fluid}

The boundary and regularity conditions of the fluid are given in Eqs. (16), (30) and (31). The quadrature analogs of the fluid boundary conditions are simply 


$$
\begin{gathered}
\sum_{j=1}^{n} A_{n j}^{(1)} P_{k}\left(R_{j}, Z\right)=0, \quad Z=Z_{1}, Z_{2}, \ldots, Z_{m}, \quad k=1,2,3,4, \ldots \\
\sum_{j=1}^{n} \bar{A}_{m j}^{(1)}\left\{\boldsymbol{P}_{k}\left(Z_{j}\right)\right\}=\{\mathbf{0}\}_{n \times 1}, \quad k=1,2,3,4, \ldots \\
\sum_{j=1}^{n} \bar{A}_{1 j}^{(1)}\left\{\boldsymbol{P}_{k}\left(Z_{j}\right)\right\}=\lambda \vartheta \Omega^{2}\left\{\boldsymbol{W}_{k}\right\}, \quad k=1,2,3,4, \ldots
\end{gathered}
$$

Similarly, the quadrature analogs of the regularity conditions are obtained as

$$
\begin{gathered}
P_{k}\left(R_{1}, Z\right)=0, \quad Z=Z_{1}, Z_{2}, \ldots, Z_{m}, \quad k=1,3,5,7, \ldots \\
\sum_{j=1}^{n} A_{1 j}^{(1)} P_{k}\left(R_{j}, Z\right)=0, \quad Z=Z_{1}, Z_{2}, \ldots, Z_{m}, \quad k=0,2,4,6, \ldots
\end{gathered}
$$

\subsection{Solution of Resulting System of Coupled Eigenvalue Equations}

Substituting Eq. (55) into Eq. (45) gives

$$
\left[\tilde{\boldsymbol{K}}_{k}^{f}\right]\left\{\boldsymbol{P}_{k}\right\}+\Omega^{2}\left[\tilde{\boldsymbol{M}}^{f}\right]\left\{\boldsymbol{P}_{k}\right\}=\Omega^{2}\left[\boldsymbol{M}^{*}\right]\left\{\boldsymbol{W}_{k}\right\}
$$

where in

$$
\left[\boldsymbol{M}^{*}\right]=\left[\begin{array}{c}
\lambda \vartheta[\boldsymbol{I}]_{n \times n} \\
{[\mathbf{0}]_{n \times n}} \\
{[\mathbf{0}]_{n \times n}} \\
\vdots \\
{[\mathbf{0}]_{n \times n}}
\end{array}\right]_{n m \times n}
$$

Eq. (34) can also be rewritten as

$$
\left[\boldsymbol{K}_{k}^{s}\right]\left\{\boldsymbol{W}_{k}\right\}-\Omega^{2}\left[\boldsymbol{M}^{s}\right]\left\{\boldsymbol{W}_{k}\right\}=-\left[\boldsymbol{K}^{*}\right]\left\{\boldsymbol{P}_{k}\right\}
$$

where

$$
\left[\boldsymbol{K}^{*}\right]=\frac{1}{\lambda \vartheta}\left[\boldsymbol{M}^{*}\right]^{T}=\left[\left[\begin{array}{lllll}
\boldsymbol{I}]_{n \times n} & {[\mathbf{0}]_{n \times n}} & {[\mathbf{0}]_{n \times n}} & \cdots & \left.[\mathbf{0}]_{n \times n}\right]_{n \times n m}
\end{array}\right.\right.
$$

As it can be seen, the added mass and stiffness matrices due to fluid-structure interaction (i.e., the matrices $\left[\boldsymbol{M}^{*}\right]$ and $\left.\left[\boldsymbol{K}^{*}\right]\right)$ have very simple forms. Now, combining Eq. (58) with Eq. (60) gives

$$
\left[\begin{array}{cc}
{\left[\tilde{\boldsymbol{K}}_{k}^{f}\right]_{n m \times n m}} & {[\mathbf{0}]_{n m \times n}} \\
{\left[\boldsymbol{K}^{*}\right]_{n \times n m}} & {\left[\boldsymbol{K}_{k}^{s}\right]_{n \times n}}
\end{array}\right]\left\{\begin{array}{l}
\left\{\boldsymbol{P}_{k}\right\}_{n m \times 1} \\
\left\{\boldsymbol{W}_{k}\right\}_{n \times 1}
\end{array}\right\}=\Omega^{2}\left[\begin{array}{cc}
-\left[\tilde{\boldsymbol{M}}^{f}\right]_{n m \times n m} & {\left[\boldsymbol{M}^{*}\right]_{n m \times n}} \\
{[\mathbf{0}]_{n \times n m}} & {\left[\boldsymbol{M}^{s}\right]_{n \times n}}
\end{array}\right]\left\{\begin{array}{l}
\left\{\boldsymbol{P}_{k}\right\}_{n m \times 1} \\
\left\{\boldsymbol{W}_{k}\right\}_{n \times 1}
\end{array}\right\}
$$

After substituting the remaining quadrature analog equations (given in Eqs. 49-52, 53, 54, 56 and 57) into Eq. (62), one can solve the resulting eigenvalue problem for the eigenvalues $\Omega$. 


\section{POTENTIAL-BASED DQM PROCEDURE}

Substituting Eqs. (26) and (29) into Eqs. (23) and (24) gives

$$
\begin{gathered}
{\left[\frac{\mathrm{d}^{4}}{\mathrm{~d} R^{4}}+\frac{2}{R} \frac{\mathrm{d}^{3}}{\mathrm{~d} R^{3}}-\frac{1+2 k^{2}}{R^{2}} \frac{\mathrm{d}^{2}}{\mathrm{~d} R^{2}}+\frac{1+2 k^{2}}{R^{3}} \frac{\mathrm{d}}{\mathrm{d} R}+\frac{k^{4}-4 k^{2}}{R^{4}}\right] W_{k}(R)+\Omega^{2} W_{k}(R)=\Omega \Theta_{k}(R, Z=0)} \\
{\left[\frac{\partial^{2}}{\partial R^{2}}+\frac{1}{R} \frac{\partial}{\partial R}-\frac{k^{2}}{R^{2}}+\frac{1}{\vartheta^{2}} \frac{\partial^{2}}{\partial Z^{2}}\right] \Theta_{k}(R, Z)-\frac{1}{c^{2}} \Omega^{2} \Theta_{k}(R, Z)=0}
\end{gathered}
$$

The procedure for discretizing Eqs. (63) and (64) and the corresponding regularity and boundary conditions is similar to that described in Section 5 . To save the space, only the resultant system of eigenvalue equations is given in this section. That is,

$$
\begin{aligned}
& \left(\Omega^{2}\left[\begin{array}{cc}
-\left[\tilde{\boldsymbol{M}}^{f}\right]_{n m \times n m} & {[\mathbf{0}]_{n m \times n}} \\
{[\mathbf{0}]_{n \times n m}} & {\left[\boldsymbol{M}^{s}\right]_{n \times n}}
\end{array}\right]-\Omega\left[\begin{array}{cc}
{[\mathbf{0}]_{n m \times n m}} & {\left[\boldsymbol{C}^{*}\right]_{n m \times n}} \\
{\left[\boldsymbol{C}^{* *}\right]_{n \times n m}} & {[\mathbf{0}]_{n \times n}}
\end{array}\right]+\left[\begin{array}{cc}
{\left[\tilde{\boldsymbol{K}}_{k}^{f}\right]_{n m \times n m}} & {[\mathbf{0}]_{n m \times n}} \\
{[\mathbf{0}]_{n \times n m}} & {\left[\boldsymbol{K}_{k}^{s}\right]_{n \times n}}
\end{array}\right]\right)\left\{\begin{array}{c}
\left\{\boldsymbol{\Theta}_{k}\right\}_{n m \times 1} \\
\left\{\boldsymbol{W}_{k}\right\}_{n \times 1}
\end{array}\right\}= \\
& \left\{\begin{array}{c}
\{\mathbf{0}\}_{n m \times 1} \\
\{\mathbf{0}\}_{n \times 1}
\end{array}\right\}
\end{aligned}
$$

where the matrices $\left[\tilde{\boldsymbol{M}}^{f}\right],\left[\boldsymbol{M}^{s}\right],\left[\tilde{\boldsymbol{K}}_{k}^{f}\right]$ and $\left[\boldsymbol{K}_{k}^{s}\right]$ are exactly the same as those defined in Section 5 . Furthermore,

$$
\begin{gathered}
{\left[\boldsymbol{C}^{*}\right]=\left[\boldsymbol{M}^{*}\right],\left[\boldsymbol{C}^{* *}\right]=\left[\boldsymbol{K}^{*}\right]} \\
\left\{\boldsymbol{\Theta}_{k}\right\}=\left\{\begin{array}{llll}
\left\{\boldsymbol{\Theta}_{k}\left(Z_{1}\right)\right\}^{T} & \left\{\boldsymbol{\Theta}_{k}\left(Z_{2}\right)\right\}^{T} & \ldots & \left.\left\{\boldsymbol{\Theta}_{k}\left(Z_{m}\right)\right\}^{T}\right\}^{T} \\
\left\{\boldsymbol{\Theta}_{k}(Z)\right\}=\left\{\Theta_{k}\left(R_{1}, Z\right)\right. & \Theta_{k}\left(R_{2}, Z\right) & \ldots & \left.\Theta_{k}\left(R_{n}, Z\right)\right\}^{T}
\end{array}\right.
\end{gathered}
$$

where in the matrices $\left[\boldsymbol{M}^{*}\right]$ and $\left[\boldsymbol{K}^{*}\right]$ are defined in Section 5 .

\section{COMPARISON OF THE TWO PROCEDURES}

It can be seen from Eq. (62) that the effect of fluid-structure interaction in pressure-based formulation is appeared in the form of added mass and stiffness matrices $\left[\boldsymbol{M}^{*}\right]$ and $\left[\boldsymbol{K}^{*}\right]$. In general, the elements of matrix $\left[\boldsymbol{K}^{*}\right]$ are not in the same order of magnitude as compared with those of matrix $\left[\boldsymbol{K}_{k}^{s}\right]$. For instance, they may be much smaller than those of matrix $\left[\boldsymbol{K}_{k}^{s}\right]$. Similarly, the order of elements of matrix $\left[\boldsymbol{M}^{*}\right]$ may be very different in magnitude as compared with that of matrix $\left[\tilde{\boldsymbol{M}}^{f}\right]$. As a result, the eigenvalue problem (62) may become ill-conditioned particularly when the dimensional differential equations of the fluid and structure are handled using the DQM.

The above-mentioned difficulty can be easily overcome by the help of potential-based formulation. This can be clearly seen from Eq. (65) wherein the effect of fluid-structure interaction is appeared in the form of added damping matrices $\left[\boldsymbol{C}^{*}\right]$ and $\left[\boldsymbol{C}^{* *}\right]$. As a result, the problem of ill- 
conditioning is fully eliminated in the potential-based formulation even if the dimensional differential equations of the problem are discretized using the DQM.

\section{NUMERICAL RESULTS AND DISCUSSION}

\subsection{Verification and Validation of Numerical Results}

To validate the proposed procedures for free vibration analysis of fluid-loaded circular plates, application is made to a numerical example given by Tariverdilo et al. (2013). Tariverdilo et al. (2013) proposed two analytical approaches, i.e., the Fourier-Bessel series method and the variational method, for asymmetric free vibration of circular plates in contact with a bounded incompressible fluid. The parameters used in their study are as follows:

$$
\varrho s=7800 \mathrm{Kg} / \mathrm{m}^{3}, a=100 \mathrm{~mm}, h=2 \mathrm{~mm}, \varrho f=1000 \mathrm{Kg} / \mathrm{m}^{3}, H=100 \mathrm{~mm}, E=210 \mathrm{GPa}, \mu=0.3
$$

where $E$ is modulus of elasticity while $\mu$ is the Poisson's ratio. Although the proposed techniques are developed for free vibration analysis of circular plates in contact with a compressible fluid, they are also available for that of circular plates in contact with an incompressible fluid if the sound speed $c_{f}$ is assumed to be infinity or the mass matrix of the fluid is assumed to be zero matrix.

Figure 2 presents the convergence of solutions for axisymmetric and asymmetric natural frequencies of the fluid-loaded circular clamped plate. The results are obtained using both the pressure-based and potential-based DQM procedures. It can be seen that the solutions of the pressure-based procedure are exactly identical to those of the potential-based procedure. Besides, both procedures predict zero value for the fundamental frequency $\left(\omega_{0,1}\right)$ of the coupled system. The existence of zero-frequency mode for Neumann problem has been discussed at length in Eftekhari and Jafari (2014) and is not repeated here again. The present results show that such phenomenon may also be observed in free vibration problem of fluid-loaded circular plates with Neumann boundary conditions.
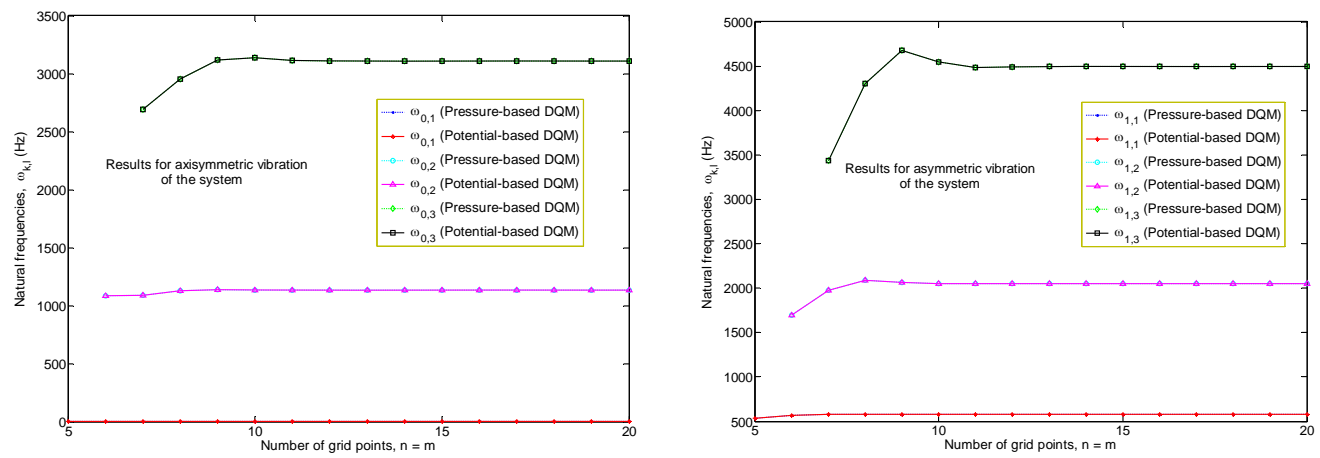

Figure 2: Convergence of axisymmetric and asymmetric natural frequencies of a fluid-loaded circular clamped plate.

On the other hand, from Figure 2 one sees that the obtained solutions show an excellent convergence behavior with increasing number grid points. Besides, a reasonably converged solution can be achieved by the proposed procedures using a considerably small number of grid points. It is clear that the accurate simulation of the higher modes of the coupled system requires a larger number of grid points. 
In Table 1, the results of present simulations (pressure-based and potential-based DQMs) are compared with the approximate analytical solutions of Tariverdilo et al. (2013). It can be seen that the results generated by the present methodologies converge very quickly and agree well with the analytical solutions of Tariverdilo et al. (2013). Besides, the present results have closer agreement with the results of the variational formulation than those of the Fourier-Bessel series. It is interesting to note that, almost in most cases, the present procedures can produce a reasonably accurate solution for natural frequencies of the system using only 11 grid points $(n=m=11)$.

On the other hand, from Table 1 one sees that the solutions of the pressure-based procedure are exactly identical to those of the potential-based procedure. As we discussed before in Eftekhari (2015), this is due to physical equivalence of the pressure-based formulation and the potential-based formulation (i.e., physical equivalence of the governing differential equations and boundary conditions for both formulations).

\begin{tabular}{|c|c|c|c|c|c|c|c|c|c|}
\hline$\omega_{\mathrm{k}, \mathrm{l}}$ & Method & $n=11^{\mathrm{a}}$ & $n=13$ & $n=15$ & $n=17$ & $n=19$ & $n=21$ & $\begin{array}{c}\text { Fourier-Bessel } \\
\text { series }^{\text {b }}\end{array}$ & $\begin{array}{l}\text { Variational } \\
\text { formulation }^{\mathrm{b}}\end{array}$ \\
\hline \multirow[t]{2}{*}{$\omega_{1,1}$} & Present $^{\mathrm{c}}$ & 578.086 & 578.086 & 578.086 & 578.086 & 578.086 & 578.086 & & \\
\hline & Present & 578.086 & 578.086 & 578.086 & 578.086 & 578.086 & 578.086 & 578.11 & 578.07 \\
\hline \multirow[t]{2}{*}{$\omega 1,2$} & Present $^{c}$ & 2051.67 & 2052.04 & 2052.03 & 2052.03 & 2052.03 & 2052.03 & & \\
\hline & Present & 2051.67 & 2052.04 & 2052.03 & 2052.03 & 2052.03 & 2052.03 & 2052.61 & 2052.03 \\
\hline \multirow[t]{2}{*}{$\omega_{2,1}$} & Present $^{c}$ & 1097.45 & 1108.05 & 1107.68 & 1107.70 & 1107.70 & 1107.70 & & \\
\hline & Present & 1097.45 & 1108.05 & 1107.68 & 1107.70 & 1107.70 & 1107.70 & 1107.84 & 1107.83 \\
\hline \multirow[t]{2}{*}{$\omega_{2,2}$} & Present $^{c}$ & 3281.02 & 3031.31 & 3066.74 & 3063.96 & 3064.08 & 3064.07 & & \\
\hline & Present & 3281.02 & 3031.31 & 3066.74 & 3063.96 & 3064.08 & 3064.07 & 3077.72 & 3086.97 \\
\hline \multirow[t]{2}{*}{$\omega_{3,1}$} & Present $^{\mathrm{c}}$ & 1752.58 & 1752.68 & 1752.68 & 1752.68 & 1752.68 & 1752.68 & & \\
\hline & Present & 1752.58 & 1752.68 & 1752.68 & 1752.68 & 1752.68 & 1752.68 & 1753.16 & 1753.27 \\
\hline \multirow[t]{2}{*}{$\omega_{3,2}$} & Present $^{\mathrm{c}}$ & 4196.92 & 4203.71 & 4203.36 & 4203.38 & 4203.38 & 4203.38 & & \\
\hline & Present & 4196.92 & 4203.71 & 4203.36 & 4203.38 & 4203.38 & 4203.38 & 4204.67 & 4203.44 \\
\hline \multirow[t]{2}{*}{$\omega 4,1$} & Present $^{c}$ & 2518.10 & 2518.66 & 2518.65 & 2518.65 & 2518.65 & 2518.65 & & \\
\hline & Present & 2518.10 & 2518.66 & 2518.65 & 2518.65 & 2518.65 & 2518.65 & 2518.79 & 2518.40 \\
\hline \multirow[t]{2}{*}{$\omega 4,2$} & Present $^{c}$ & 5470.73 & 5474.10 & 5474.04 & 5474.04 & 5474.04 & 5474.04 & & \\
\hline & Present & 5470.73 & 5474.10 & 5474.04 & 5474.04 & 5474.04 & 5474.04 & 5476.53 & 5475.36 \\
\hline
\end{tabular}

Table 1: Convergence and accuracy of asymmetric natural frequencies $(\omega k, l(\mathrm{~Hz}))$ of a fluid-loaded circular clamped plate. ${ }^{\mathrm{a}} m=n,{ }^{\mathrm{b}}$ Tariverdilo et al. (2013), ${ }^{\mathrm{c}}$ Solutions of pressure-based DQM, ${ }^{\mathrm{d}}$ Solutions of potential-based DQM.

Finally, it should be noted that the resulting eigenvalue problems of the proposed procedures are found to be well-conditioned and thus they can be easily and directly solved using various standard eigenvalue solvers. But the resultant matrices of analytical solutions of Tariverdilo et al. (2013) were reported to be highly ill-conditioned. This can lead to large errors in the calculation of the eigenvalues particularly in the calculation of higher modes of the coupled system. Tariverdilo et al. (2013) mentioned that such an eigenvalue problem can only be solved by the help of precondi- 
tioner algorithms such as the diagonal scaling method. Therefore, it can be concluded that the proposed procedures are superior over the analytical formulations of Tariverdilo et al. (2013) in terms of accuracy and reliability. Most importantly, the higher modes of the system can be easily and accurately computed by the proposed procedures without adopting any preconditioner algorithm.

\subsection{Free Vibration of Circular Plates in Contact with a Bounded Incompressible Fluid}

It can be seen from Section 3 that the natural frequencies of the coupled system in the case of incompressible fluid can be described by means of the following dimensionless parameters

$$
\lambda=\frac{\varrho_{f} a}{\varrho_{s} h}, \vartheta=\frac{H}{a}
$$

where $\lambda$ is the mass ratio and $\vartheta$ is the slenderness ratio. Table 2 gives the dimensionless asymmetric natural frequencies $\left(\Omega_{k, l}=\omega_{k, l} a^{2} \sqrt{\varrho_{s} h / D}\right)$ of the coupled system for different values of $\lambda$ and $\vartheta$. It can be seen from Table 2 that the natural frequencies of the system decrease as the value of the parameter $\lambda$ increases. Besides, they increase as the value of $\vartheta$ increases. Note that the solutions of the pressure-based DQM are found to be exactly identical to those of the potential-based DQM.

The variations of dimensionless axisymmetric natural frequencies $\left(\Omega_{k, l}=\omega_{k, l} a^{2} \sqrt{\varrho_{s} h / D}\right)$ of the fluid-loaded circular clamped plate with $\vartheta$ are shown in Figure 3 for different values of $\lambda$. Since the value of the fundamental frequency of the coupled system (i.e., $\Omega_{0,1}$ ) is zero, the results are shown for $\Omega_{k, l}>$ $\Omega_{0,1}$. It can be seen from Figure 3 that the natural frequencies of the system are initially increasing with increasing the value of $\vartheta$ and after a certain value of $\vartheta$ (say, $\vartheta^{*}$ ) they are remaining constant. The value of $\vartheta^{*}$ is almost independent of values of $\lambda$, but it decreases as the mode number increases.

\begin{tabular}{cccccccc}
\hline \hline$\vartheta$ & $\Omega_{k, l}$ & $\lambda=1$ & $\lambda=5$ & $\lambda=10$ & $\lambda=15$ & $\lambda=20$ & $\lambda=25$ \\
\hline 0.25 & $\Omega_{1,1}$ & 16.2327 & 9.9032 & 7.4092 & 6.1735 & 5.4025 & 4.8630 \\
& $\Omega_{1,2}$ & 53.7736 & 41.1425 & 33.6662 & 29.2149 & 26.1681 & 23.9127 \\
& $\Omega_{1,3}$ & 111.2275 & 92.3947 & 79.0128 & 70.2162 & 63.8418 & 58.9437 \\
& $\Omega_{1,4}$ & 188.4292 & 163.2182 & 143.3200 & 129.3945 & 118.9007 & 110.6164 \\
0.65 & $\Omega_{1,1}$ & 17.9172 & 12.1703 & 9.4040 & 7.9389 & 6.9970 & 6.3264 \\
& $\Omega_{1,2}$ & 55.3268 & 43.3404 & 35.7823 & 31.1903 & 28.0137 & 25.6465 \\
& $\Omega_{1,3}$ & 112.6166 & 94.1575 & 80.6896 & 71.7889 & 65.3207 & 60.3410 \\
& $\Omega_{1,4}$ & 189.7289 & 164.7183 & 144.6984 & 130.6702 & 120.0930 & 111.7395 \\
& $\Omega_{1,1}$ & 18.1699 & 12.5842 & 9.7915 & 8.2907 & 7.3192 & 6.6248 \\
& $\Omega_{1,2}$ & 55.5570 & 43.6753 & 36.0879 & 31.4660 & 28.2657 & 25.8797 \\
& $\Omega_{1,3}$ & 112.8366 & 94.4495 & 80.9484 & 72.0198 & 65.5306 & 60.5347 \\
& $\Omega_{1,4}$ & 189.9426 & 164.9851 & 144.9296 & 130.8748 & 120.2781 & 111.9098 \\
\hline \hline
\end{tabular}

Table 2: Dimensionless asymmetric natural frequencies of a fluid-loaded circular clamped plate. Note that the solutions are obtained using both the pressure-based and potential-based DQMs with $n=21$ and $m=17$. 

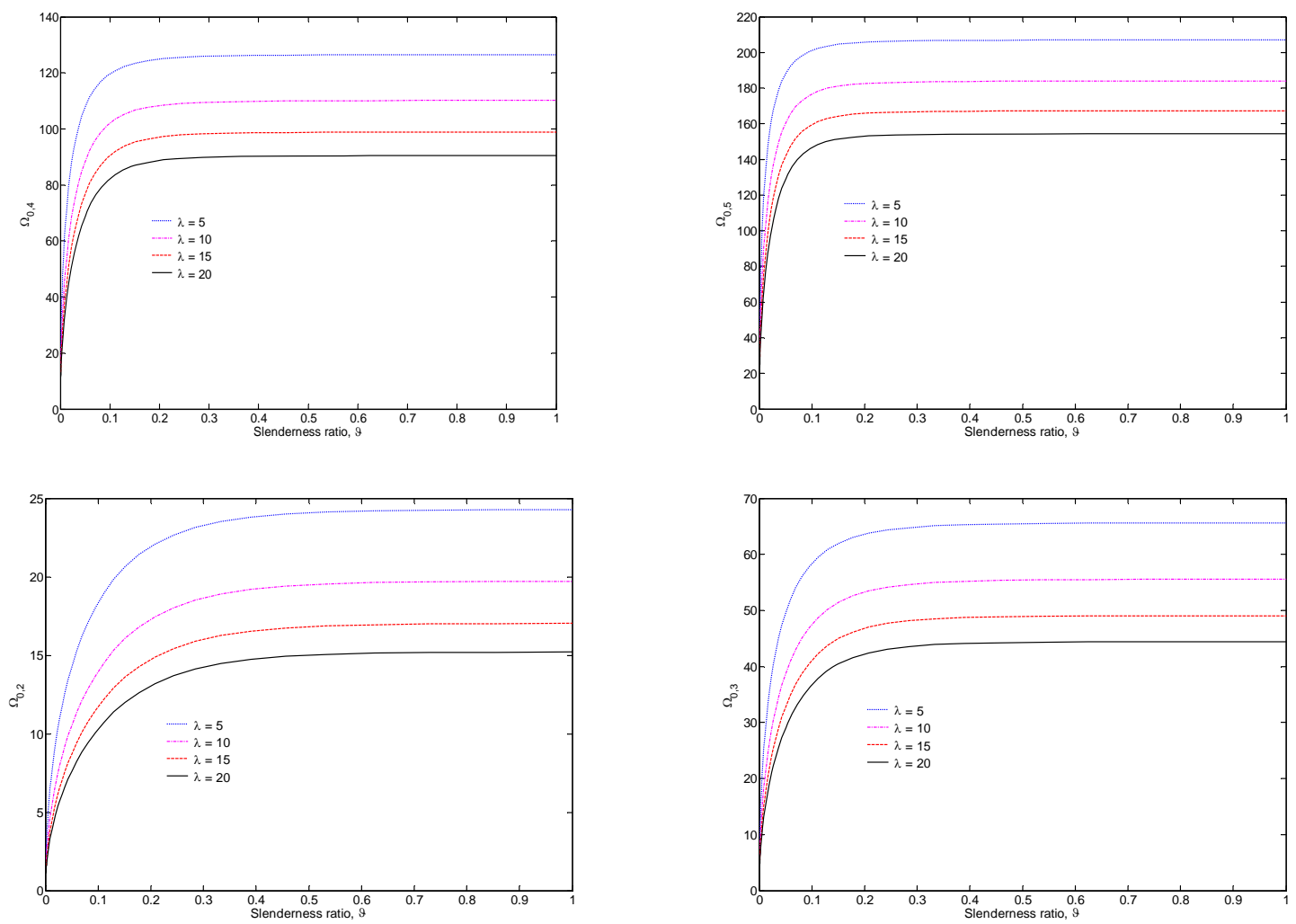

Figure 3: Variations of axisymmetric natural frequencies of a fluid-loaded circular clamped plate with $\vartheta$ for different values of $\lambda$.

Figure 4 presents the variations of dimensionless axisymmetric natural frequencies of the fluidloaded circular clamped plate with $\lambda$ for different values of $\vartheta$. Note that the results are shown for $\Omega_{k, l}>\Omega_{0,1}$. As it can be seen, the natural frequencies of the system are initially decreasing with increasing value of $\lambda$ and after a certain value of $\lambda$ (say, $\lambda^{*}$ ) they are remaining constant. The value of $\lambda^{*}$ is almost independent of values of $\vartheta$, but it increases as the mode number increases. On the other hand, from Figure 4 one sees the curve for $\vartheta=0.1$ approaches to the one for $\vartheta=0.5$ as the mode number increases. This implies that the higher modes of the coupled system are less influenced by the values of the parameter $\vartheta$ (this can also be clearly observed from the numerical results presented in Figure 3).

The numerical results for asymmetric natural frequencies of the coupled system are shown in Figures 5 and 6 . Note that the numerical results are shown for $\Omega_{1,1}$ and $\Omega_{1,4}$. As it can be seen, the behavior of results in this case (asymmetric vibration) is very similar to that of the axisymmetric vibration. 

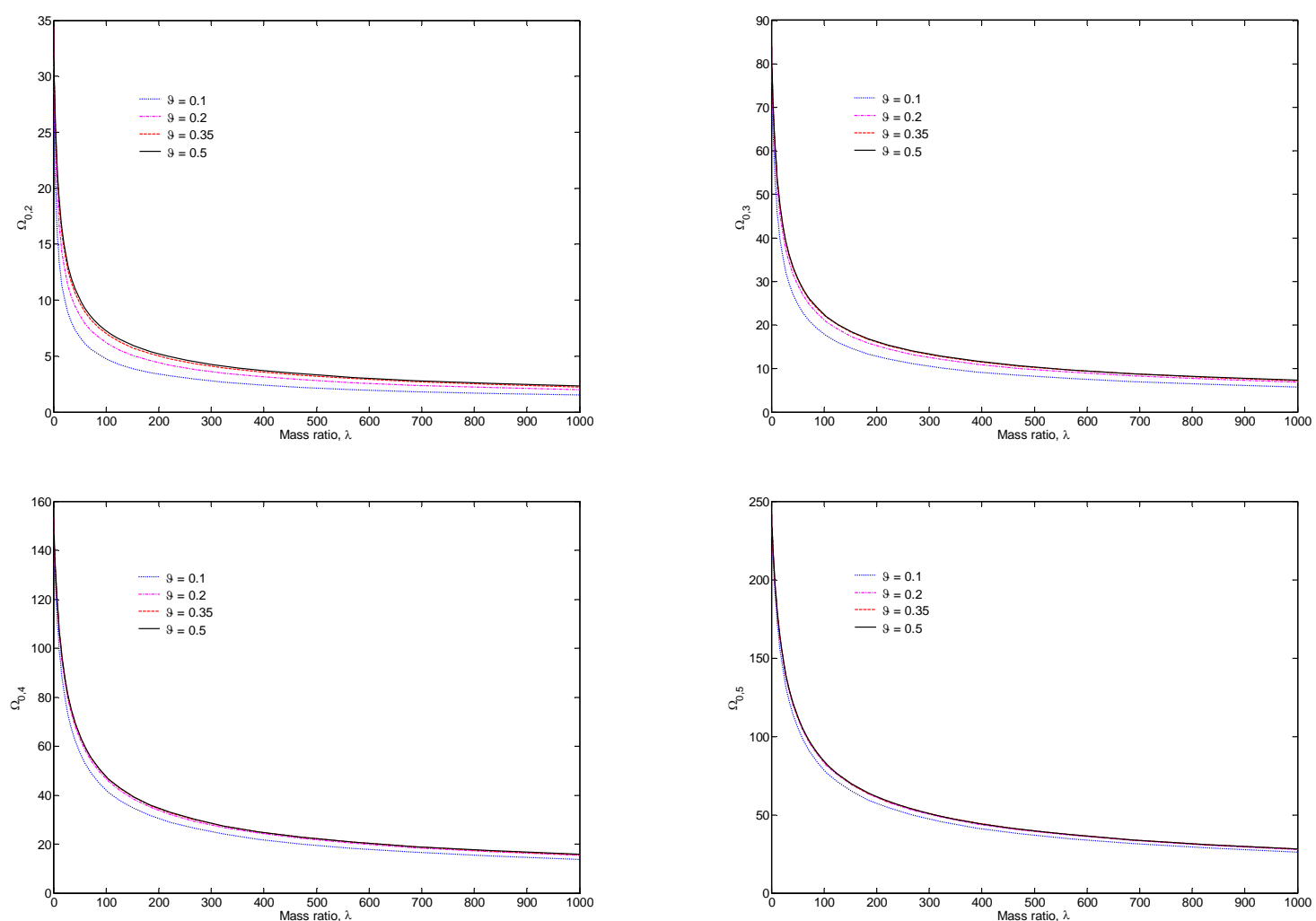

Figure 4: Variations of axisymmetric natural frequencies of a fluid-loaded circular clamped plate with $\lambda$ for different values of $\vartheta$.
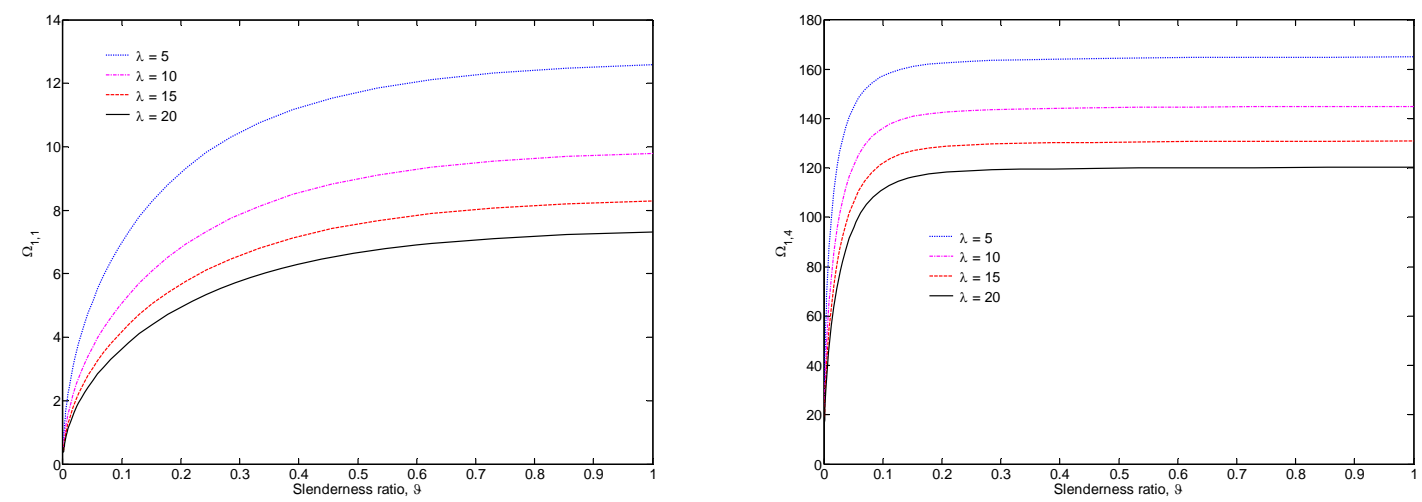

Figure 5: Variations of asymmetric natural frequencies of a fluid-loaded circular clamped plate with $\vartheta$ for different values of $\lambda$. 

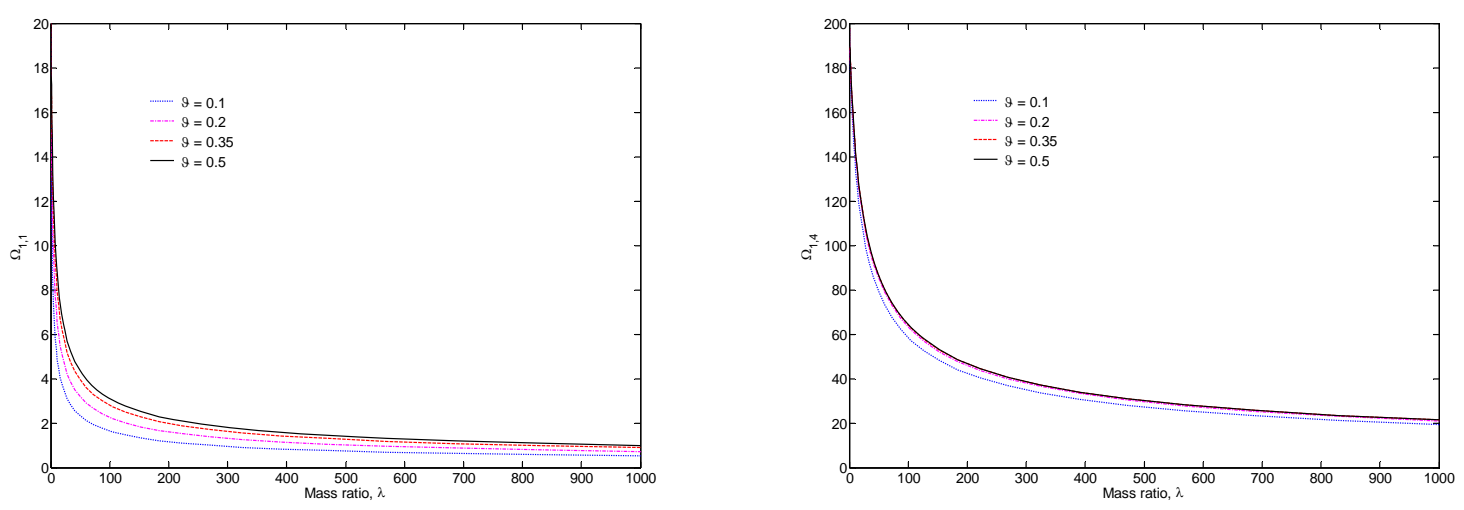

Figure 6: Variations of asymmetric natural frequencies of a fluid-loaded circular clamped plate with $\lambda$ for different values of $\vartheta$.

\subsection{Free Vibration of Circular Plates in Contact with a Bounded Compressible Fluid}

In Section 3 we showed that the natural frequencies of the coupled system in the case of compressible fluid can be expressed in terms of the following dimensionless parameters

$$
\lambda=\frac{\varrho_{f} a}{\varrho_{s} h}, \vartheta=\frac{H}{a}, \quad c=\frac{c_{f}}{c_{s}}
$$

where $c_{f}$ is the sound speed in fluid, $c_{s}$ is the sound speed in structure (circular plate) and $c$ is the speed ratio. Table 3 gives the dimensionless asymmetric natural frequencies $\left(\Omega_{k, l}=\omega_{k, l} a^{2} \sqrt{\varrho_{s} h / D}\right)$ of the coupled system for different values of $\lambda, \vartheta$ and $c$. Note that the numerical results are obtained using both the pressure-based and potential-based DQMs and are given for $\Omega_{1,1}$ and $\Omega_{1,3}$.

It can be seen from Table 3 that the natural frequencies of the system are increasing as the value of the parameter c increases. Besides, they approach to a constant value as the magnitude of this parameter increases. Again, the solutions of the pressure-based DQM are found to be exactly identical to those of the potential-based DQM. On the other hand, by comparing these results with those of Table 2, one sees the results of compressible fluid model approach to those of the incompressible fluid model as the value of $c$ increases. Furthermore, the lower modes of the system are less influenced by compressibility of the fluid as compared with the higher modes of the system.

The effects of parameters $\lambda$ and $\vartheta$ on free vibration of the coupled system have been studied in Section 8.2. Therefore, in this section we only investigate the effects of compressibility of the fluid on free vibration of the system. Theoretically, when speed ratio $(c)$ approaches to infinity while the other parameters of the problem are fixed, the solutions of the compressible fluid model should approach to those of the incompressible fluid model. To investigate this, the axisymmetric and asymmetric natural frequencies of the coupled system are plotted against $c$ in Figures 7 and 8 . These results are obtained using $\lambda=\vartheta=1$.

It can be seen from Figures 7 and 8 that the natural frequencies of the coupled system are initially increasing with increasing the magnitude of $c$ and after a certain value of $c$ (say, $c^{*}$ ) they are 
remaining the same. Note that the higher modes of the coupled system may have several $c^{*}$ (in general, the $k$ th mode of the system has $k$ number of $c^{*}$ ). But when the speed ratio is large enough (i.e., when $c=c^{*}$ ), the natural frequencies of the system do not change by increasing the magnitude of $c$. The magnitude of $c^{* f}$ is found to be dependent on the mode number and increases as the mode number increases. This implies that the higher modes of the coupled system are more affected by compressibility of the fluid.

\begin{tabular}{|c|c|c|c|c|c|c|c|}
\hline$c$ & $\vartheta$ & $\Omega_{k, l}$ & $\lambda=1$ & $\lambda=5$ & $\lambda=10$ & $\lambda=15$ & $\lambda=20$ \\
\hline \multirow[t]{4}{*}{50} & 0.25 & $\Omega_{1,1}$ & 16.1463 & 9.8667 & 7.3921 & 6.1632 & 5.3954 \\
\hline & & $\Omega_{1,3}$ & 103.5712 & 90.0745 & 77.6572 & 69.2687 & 63.1257 \\
\hline & 1.0 & $\Omega_{1,1}$ & 18.1256 & 12.5491 & 9.7714 & 8.2777 & 7.3099 \\
\hline & & $\Omega_{1,3}$ & 97.1864 & 90.9275 & 79.1996 & 70.8643 & 64.6812 \\
\hline \multirow[t]{4}{*}{100} & 0.25 & $\Omega_{1,1}$ & 16.2113 & 9.8941 & 7.4049 & 6.1709 & 5.4008 \\
\hline & & $\Omega_{1,3}$ & 110.3537 & 91.8885 & 78.6926 & 69.9872 & 63.6669 \\
\hline & 1.0 & $\Omega_{1,1}$ & 18.1591 & 12.5755 & 9.7865 & 8.2875 & 7.3169 \\
\hline & & $\Omega_{1,3}$ & 112.3034 & 93.9600 & 80.6144 & 71.7757 & 65.3424 \\
\hline \multirow[t]{4}{*}{1000} & 0.25 & $\Omega_{1,1}$ & 16.2325 & 9.9031 & 7.4092 & 6.1735 & 5.4025 \\
\hline & & $\Omega_{1,3}$ & 111.2203 & 92.3899 & 79.0096 & 70.2140 & 63.8401 \\
\hline & 1.0 & $\Omega_{1,1}$ & 18.1698 & 12.5841 & 9.7914 & 8.2907 & 7.3192 \\
\hline & & $\Omega_{1,3}$ & 112.8326 & 94.4451 & 80.9453 & 72.0175 & 65.5287 \\
\hline
\end{tabular}

Table 3: Dimensionless asymmetric natural frequencies of a fluid-loaded circular clamped plate. Note that the solutions are obtained using both the pressure-based and potential-based DQMs with $n=21$ and $m=17$.
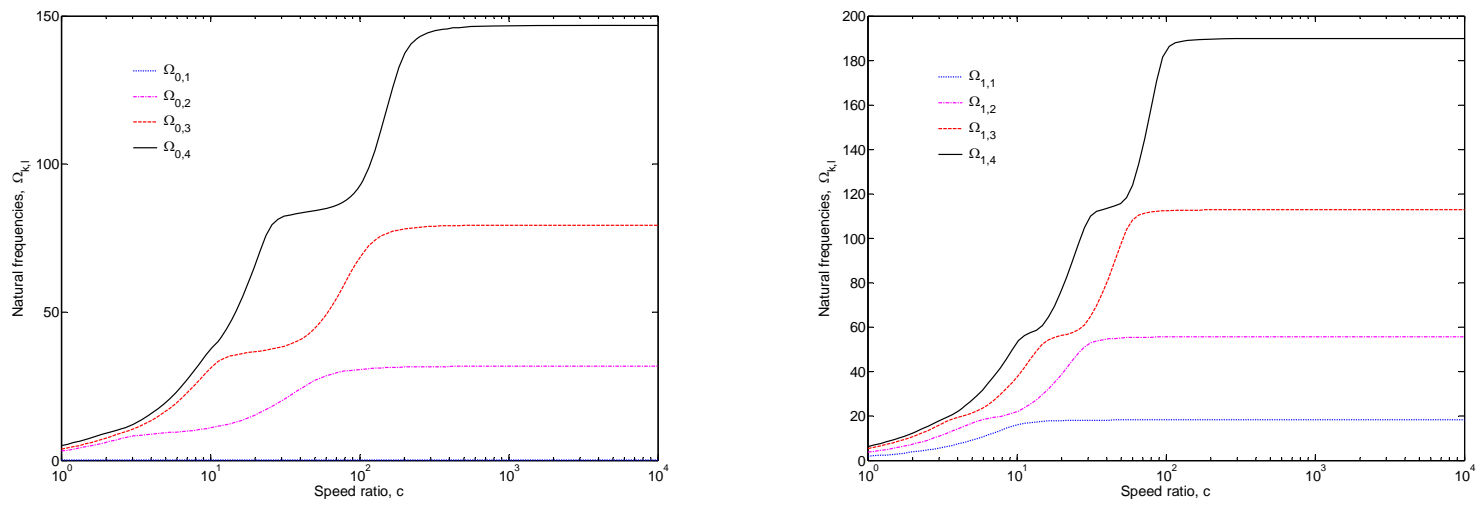

Figure 7: Variations of axisymmetric and asymmetric natural frequencies of a fluid-loaded circular clamped plate with $c(\lambda=\vartheta=1)$. 

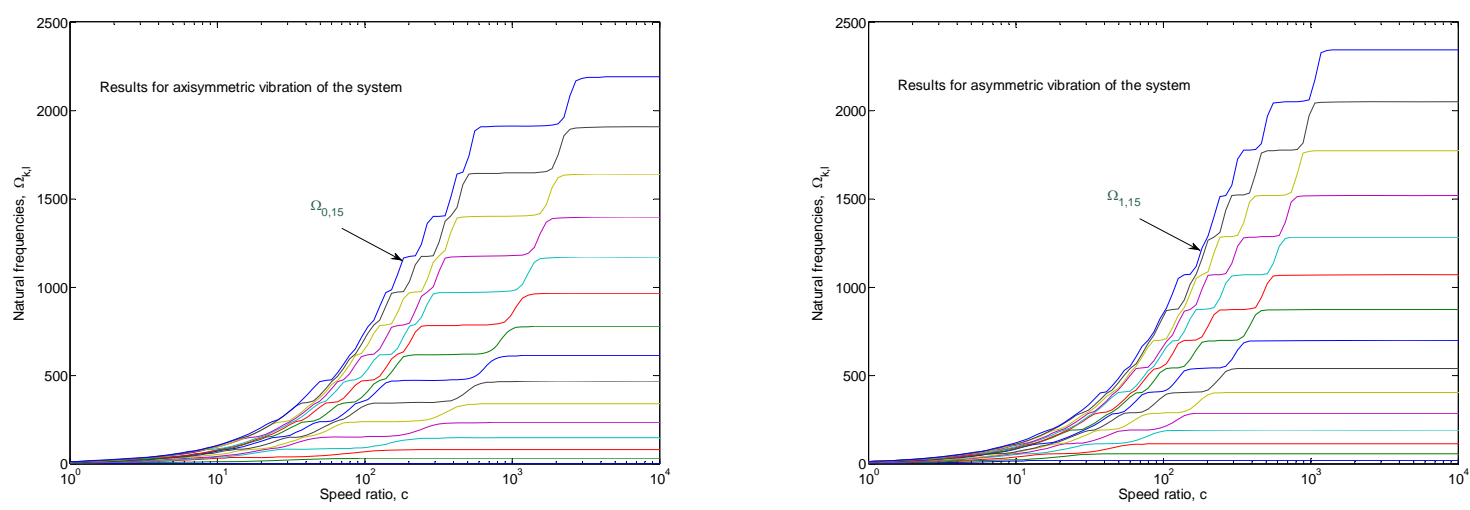

Figure 8: Variations of higher-order natural frequencies of a fluid-loaded circular clamped plate with $c(\lambda=\vartheta=1)$.

Note that $\Omega_{0,1} \leq \Omega_{k, l} \leq \Omega_{0,15}$ for the case of axisymmetric vibration of the system and $\Omega_{1,1} \leq \Omega_{k, l} \leq \Omega_{1,15}$ for the case of asymmetric vibration of the system.

\section{CONCLUSIONS}

Tow general procedures based on the DQM are developed to study the free vibration of circular plates in contact with fluid. The accuracy and reliability of the proposed procedures are examined through numerical experiments. Three new dimensionless parameters and variables are also introduced for the free vibration of the coupled system. The influences of these parameters on dynamic behavior of the system are studied.

The main advantages of the proposed procedures are their simplicity and high accuracy. The proposed procedures can also produce highly accurate converged results for natural frequencies of the coupled system by using a considerably small number of grid points. Besides, the resulting matrices of the proposed procedures are found to be well-conditioned and thus there is no need to use any preconditioner algorithm to solve the resultant eigenvalue problems.

\section{References}

Amabili, M., Pasqualini, A., Dalpiaz, G. (1995). Natural frequencies and modes of free-edge circular plates vibrating in vacuum or in contact with liquid. J Sound Vib 188: 685 - 699.

Amabili, M., Kwak, M.K. (1999). Vibration of circular plates on a free fluid surface: effect of surface waves. J Sound Vib 226(3): 407-424.

Askari, E., Daneshmand, F. (2010). Free vibration of an elastic bottom plate of a partially fluid-filled cylindrical container with an internal body. Eur J Mech A-Solid 29(1): 68-80.

Askari, E., Jeong, K.-H., Amabili, M. (2013). Hydroelastic vibration of circular plates immersed in a liquid-filled container with free surface. J Sound Vib 332: 3064 -3085.

Bauer, H.F. (1995). Coupled frequencies of a liquid in a circular cylindrical container with elastic liquid surface cover. J Sound Vib 180(5): 689-704.

Bellman, R.E., Casti, J. (1971). Differential quadrature and long term integration. J Math Anal Appl 34: $235-238$.

Bellman, R.E., Kashef, B.G., Casti, J. (1972). Differential quadrature: A technique for the rapid solution of nonlinear partial differential equations. J Comput Phys 10: 40-52. 
Bert, C.W., Malik, M. (1996). Differential quadrature method in computational mechanics: A review. ASME Appl Mech Rev 49: 1-28.

Chiba, M. (1994). Axisymmetric free hydroelastic vibration of a flexural bottom plate in a cylindrical tank supported on an elastic foundation. J Sound Vib 169: 387-394.

Ergin, A., Ugurlu, B. (2004). Hydroelastic analysis of fluid storage tanks by using a boundary integral equation method. J Sound Vib 275: 489-513.

Eftekhari, S.A., Jafari, A.A. (2013). Numerical solution of general boundary layer problems by the method of differential quadrature. Sci Iran B 20(4):1278-1301.

Eftekhari, S.A., Jafari, A.A. (2014). A mixed modal-differential quadrature method for free and forced vibration of beams in contact with fluid. Meccanica 49:535-564.

Eftekhari, S.A. (2016). Pressure-based and potential-based mixed Ritz-differential quadrature formulations for free and forced vibration of Timoshenko beams in contact with fluid. Meccanica 51:179-210.

Fantuzzi, N., Tornabene, F., Viola, E., Ferreira, A.J.M. (2014). A strong formulation finite element method (SFEM) based on RBF and GDQ techniques for the static and dynamic analyses of laminated plates of arbitrary shape. Meccanica 49(10):2503-2542.

Gorman, D.J., Horáček, J. (2007). Analysis of the free vibration of a coupled plate/fluid interacting system and interpretation using sub-system modal energy. Eng Struct 29: 754-762.

Gorman, D.J., Trendafilova, I., Mulholland, A.J., Horáček, J. (2008). Vibration analysis of a circular plate in interaction with an acoustic cavity leading to extraction of structural modal parameters. Thin-Walled Struct 46: 878-886.

Jeong, K.H. (2003). Free vibration of two identical circular plates coupled with bounded fluid. J Sound Vib 260: 653670 .

Jeong, K.-H., Kim, K.-J. (2005). Hydroelastic vibration of a circular plate submerged in a bounded compressible fluid. J Sound Vib 283: 153-172.

Jeong, K.-H., Lee, G.-M., Kim, T.-W. (2009). Free vibration analysis of a circular plate partially in contact with a liquid. J Sound Vib 324: 194-208.

Kim, Y.-W., Lee, Y.-S. (2005). Coupled vibration analysis of liquid-filled rigid cylindrical storage tank with an annular plate cover. J Sound Vibr 279: 217-235.

Koohkan, M.R., Attarnejad, R., Nasseri, M. (2010). Time domain analysis of dam-reservoir interaction using coupled differential quadrature and finite difference methods. Eng Comput 27(2):280-294.

Kwak, M.K., Kim, K.C. (1991). Axisymmetric vibration of circular plates in contact with fluid. J Sound Vib 146(3): 381-389.

Kwak, M.K. (1997). Hydroelastic vibration of circular plates. J Sound Vib 201(3): 293-303.

Kwak, M.K., Han, S.-B. (2000). Effect of fluid depth on the hydroelastic vibration of free-edge circular plate. J Sound Vib 230(1): 171-185.

Liu, G.R., Wu, T.Y. (2001). An application of the generalized differential quadrature rule in Blasius and Onsager equations. Int J Numer Meth Eng 52:1013-1027.

Quan, J.R., Chang, C.T. (1989). New insights in solving distributed system equations by the quadrature methods, Part I: analysis. Comput Chem Eng 13:779-788.

Rao, S.S. (2007). Vibration of Continuous Systems. John Wiley \& Sons, Inc. (New Jersey).

Shafiee, A.A., Daneshmand, F., Askari, E., Mahzoon, M. (2014). Dynamic behavior of a functionally graded plate resting on Winkler elastic foundation and in contact with fluid. Struct Eng Mech 50(1): 53-71.

Shu, C., Richards, B.E. (1992). Parallel simulation of incompressible viscous flows by generalized differential quadrature. Comput Syst Eng 3: 271-281. 
Shu, C., Chew, Y.T., Richards, B.E. (1995). Generalized differential-integral quadrature and their application to solve boundary layer equations. Int J Numer Meth Fluids 21:723-733.

Shu, C., Chew, Y.T. (1999). Solution of Helmholtz equation by differential quadrature method. Comput Meth Appl Mech Eng 175: 203-212.

Shu, C. (2000). Differential Quadrature and Its Application in Engineering. Springer (New York).

Tariverdilo, S., Shahmardani, M., Mirzapour, J., Shabani, R. (2013). Asymmetric free vibration of circular plate in contact with incompressible fluid. Appl Math Model 37: 228-239.

Tornabene, F., Fantuzzi, N., Ubertini, F., Viola, E. (2015). Strong formulation finite element method: a survey. ASME Appl Mech Rev 67:020801.

Wu, T.Y., Liu, G.R. (2001). Free vibration analysis of circular plates with variable thickness by the generalized differential quadrature rule. Int J Solids Struct 38: 7967-7980.

Wu, T.Y., Wang, Y.Y., Liu, G.R. (2002). Free vibration analysis of circular plates using generalized differential quadrature rule. Comput Meth Appl Mech Eng 191:5365-5380. 